\title{
The Effect of SARS-COV-2 Viruses on the Function of Different Organs, Especially the Nervous System
}

\author{
Ali-Mohammad Khanizadeh ${ }^{1,2}$, Maryam Ejlali ${ }^{3}$, Fariba Karimzadeh ${ }^{3 *}$ \\ ${ }^{1}$ Shefa Neuroscience Research Center, Khatam Alanbia Hospital, Tehran, Iran \\ ${ }^{2}$ Department of Physiology, Iran University of Medical Sciences, Tehran, Iran \\ ${ }^{3}$ Cellular and Molecular Research Center, Iran University of Medical Sciences, Tehran, Iran
}

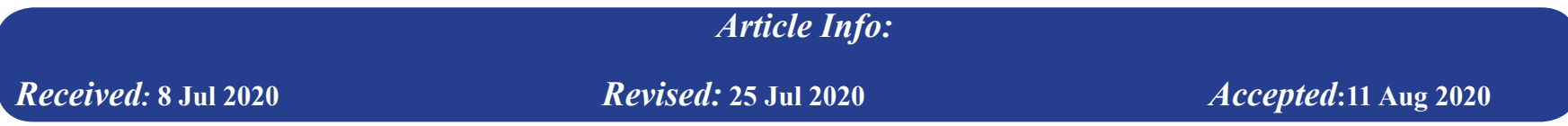

\section{ABSTRACT}

Introduction: The new coronavirus (SARS-CoV-2) has recently emerged around the world as a pandemic. The disease has various symptoms such as cough, dyspnea, and fever. The disease causes failure in different organs, including the respiratory, gastrointestinal, kidney, and central nervous systems. The exact pathobiology of the virus is incompletely known. In this s tudy, by reviewing previous evidence and studies, we have reviewed the possible mechanisms and ways of contracting the central nervous system. Conclusion: One way for coronavirus to enter the nervous system is through the angiotensin-converting enzyme 2 receptor. This receptor express in different tissues and the severity of virus infection are directly related to the amount of receptor in the tissues. Viruses can enter the brain directly through neuronal and blood circulation pathways. Common symptoms of the central nervous system during 'SARS-CoV-2 infection include dizziness, headaches, weakness, decreased consciousness, and paresthesia. Encephalitis, demyelination, neuropathy, and stroke have been reported in association with SARS-CoV-2. Invasion of the medullary cardiorespiratory control center by SARS-CoV-2 may contribute to the refractory respiratory failure observed in severe COVID-19 infected patients.

\section{Key words:}

1. Coronavirus

2. Nervous System 3. Inflammation The neuro-invasive and neurotrophic effects of coronaviruses might be related to the entry of pro-inflammatory cytokines from the peripheral circulatory system during the cytokine storm, as well as the production of these cytokines by microglia and astrocytes.

*Corresponding Author: Fariba Karimzadeh

E-mail: Karimzade.f@iums.ac.ir 
تأثير ويروس هاى سارس - كوويد - بر عملكرد ار Fانهاى مختلف بهويزه سيستم عصبى

\author{
على محمد خانى زاده ‘'، مريم اجلالى"، فريبا كريم زاده"*" \\ 'مركز تحقيقات علوم اعصاب شفا، بيمارستان خاتمالانبيا، تهران ايران \\ كَّروه فيزيولوزى، دانشعاه علوم يزشكى ايران، تهران، ايران \\ "مركز تحقيقات سلولى و مولكولى، دانشكاه علوم يزشكى ايران، تهران، ايران
}

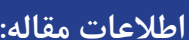

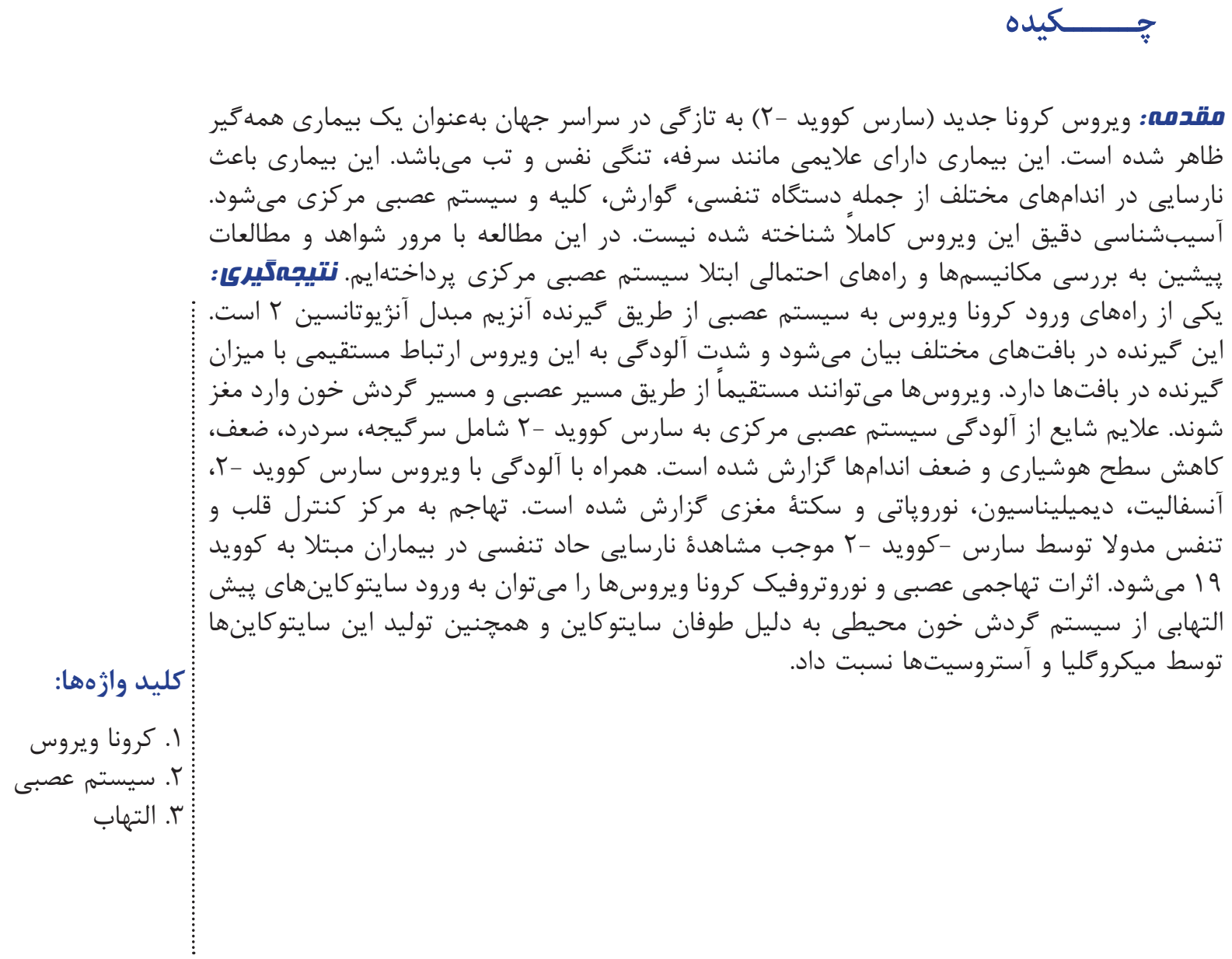

" نويسنده مسئول: فريبا كريم زاده

آدرس الكترونيكى: Karimzade.f@iums.ac.ir 


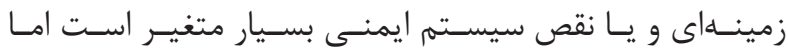

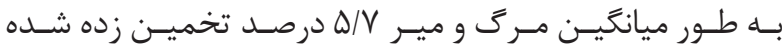

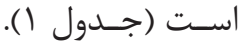

\section{بيامدهاى ابتلا به سارس كوويد -}

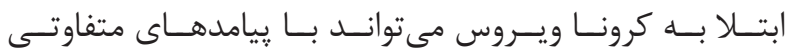

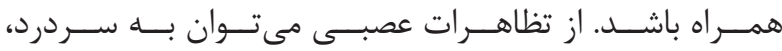

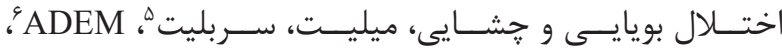

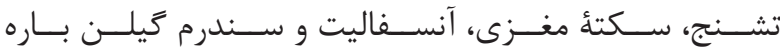

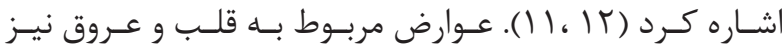

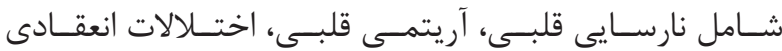

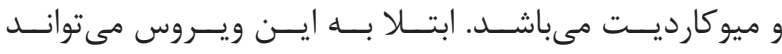

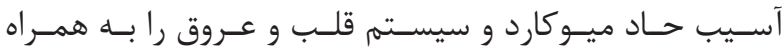

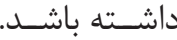

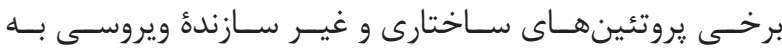

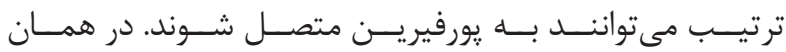

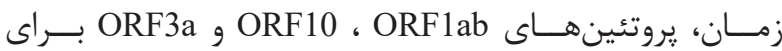

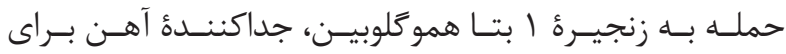

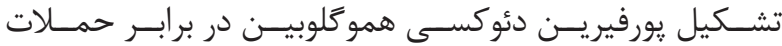

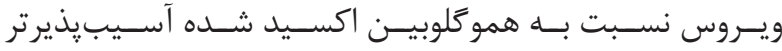

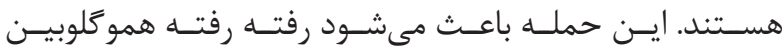

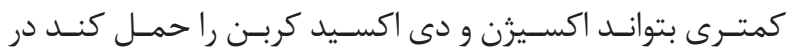

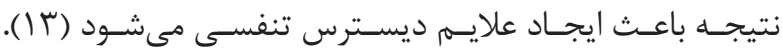

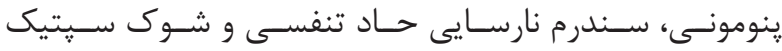

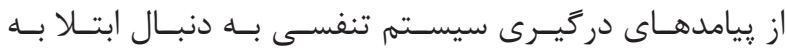

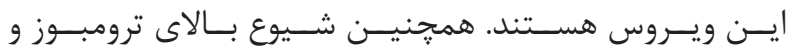

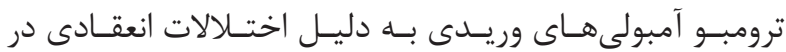

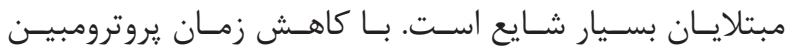

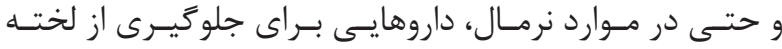

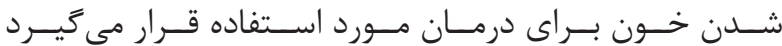

$(|f-| V)$

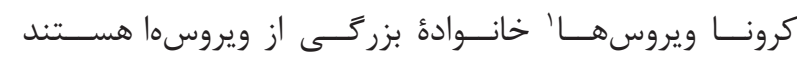

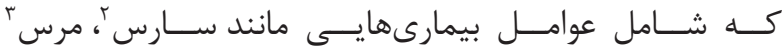

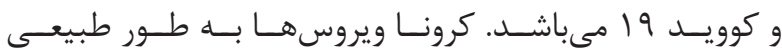

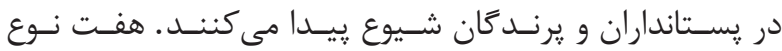

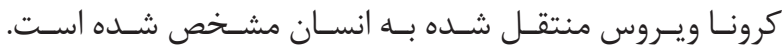

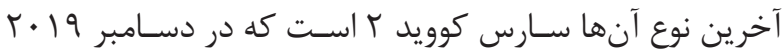

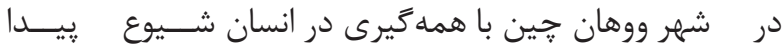

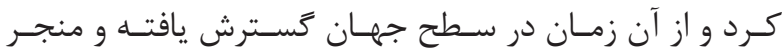

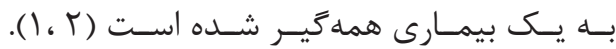

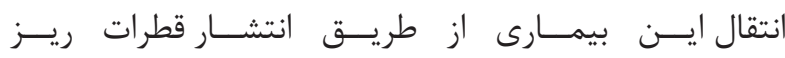

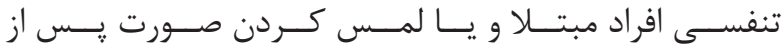

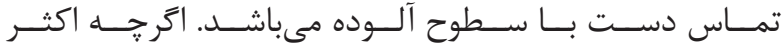

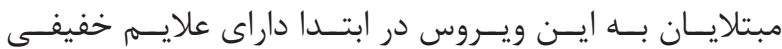

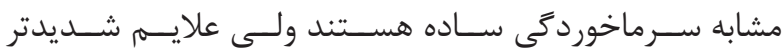

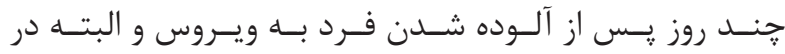

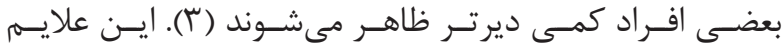

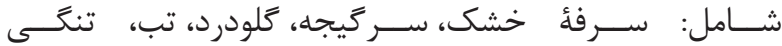

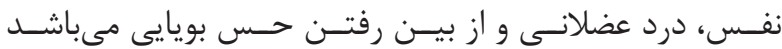

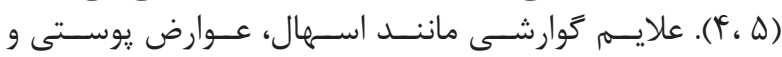

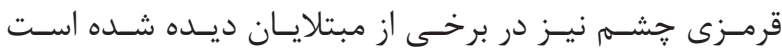

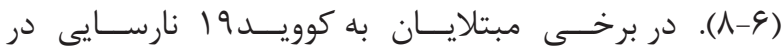

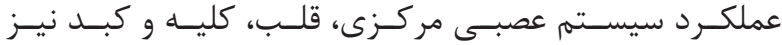

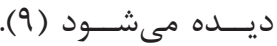
عوامل خطر

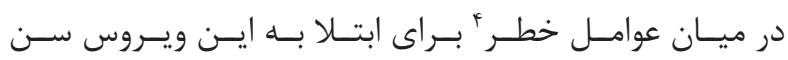

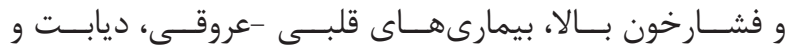

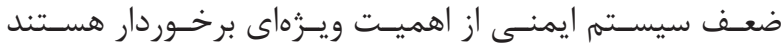

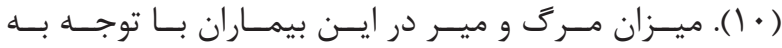

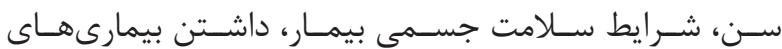

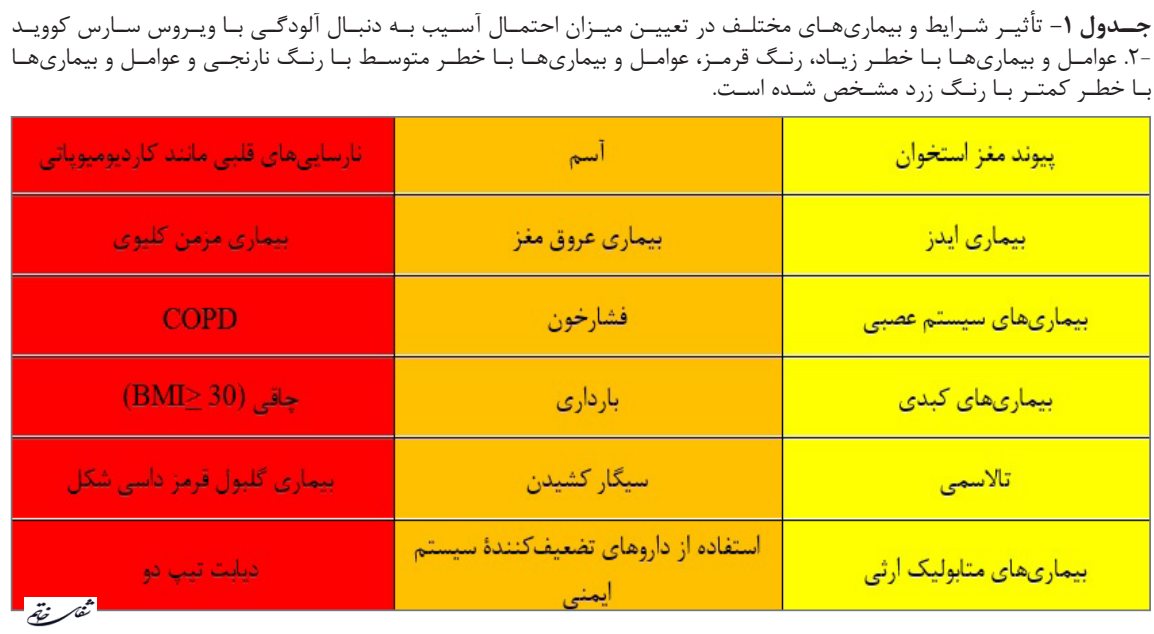

${ }^{1}$ Corona virus

${ }^{2}$ Sars

${ }^{3}$ Mers
${ }^{4}$ Risk factors

${ }^{5}$ Cerebellitis

${ }^{6}$ Acute disseminated encephalomyelitis 


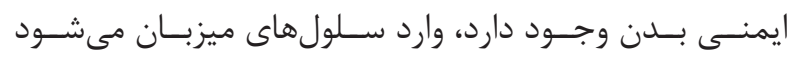
دوى (YG، TV)

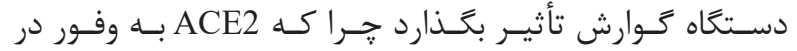

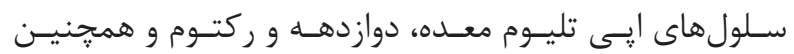

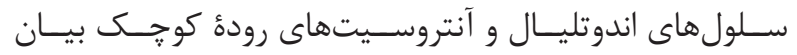

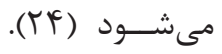

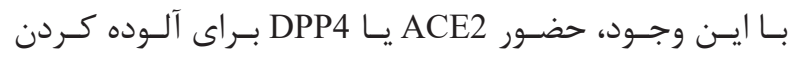

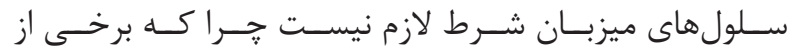

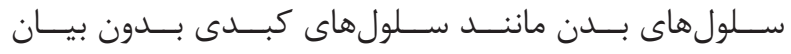
ACE2

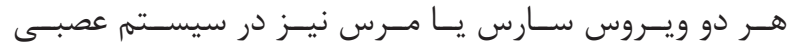

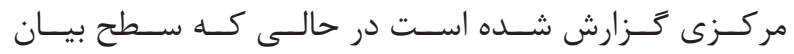

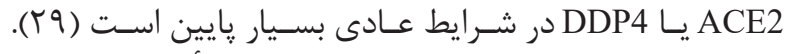

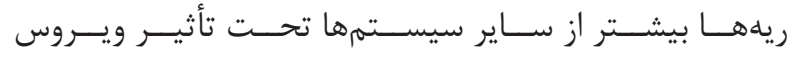

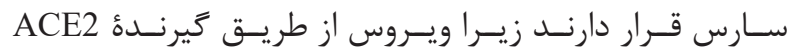

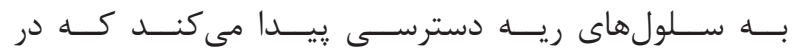

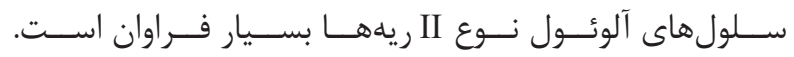

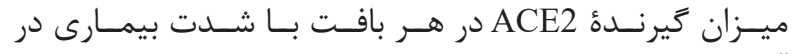

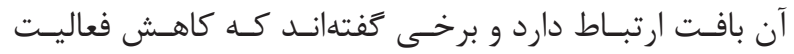
ACE2

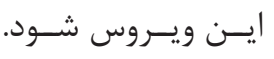

كرونا ويروسها و سيستمر عصبى مركزى

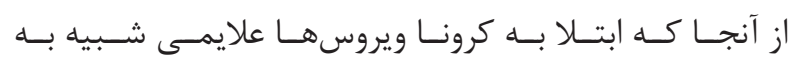

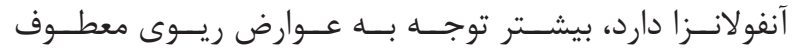

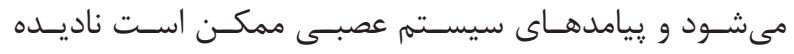

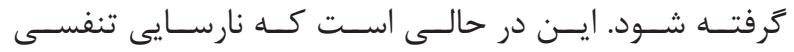

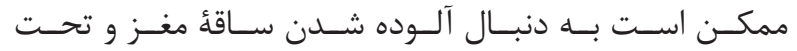

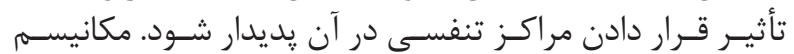

كاهـش عملكــرد كليـهـ و افزايـش كراتنيـن نيـز در مبتلايــان

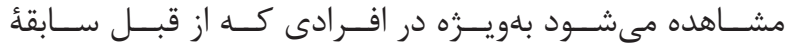

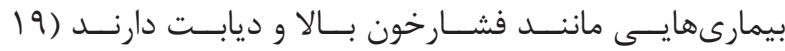

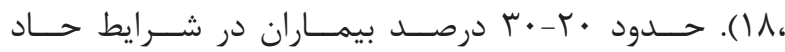

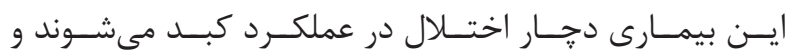

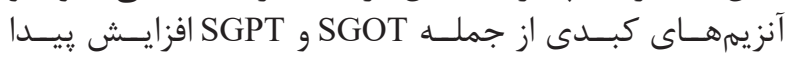

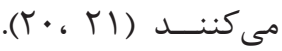

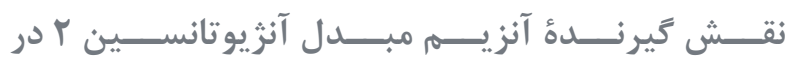

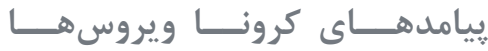

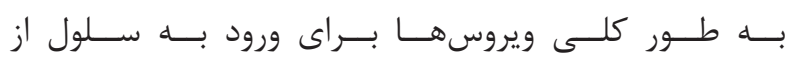

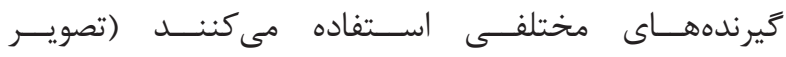

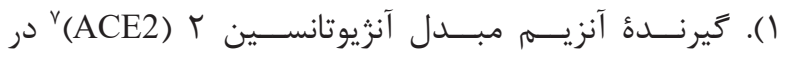

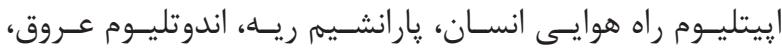

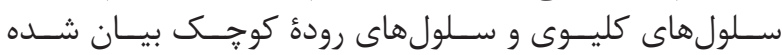

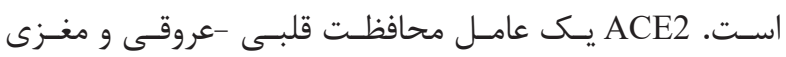

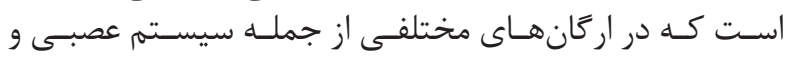

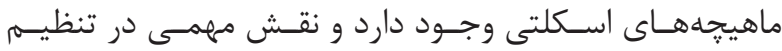

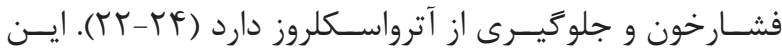

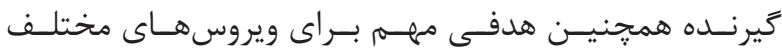

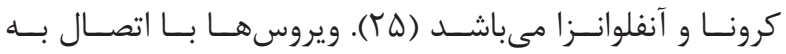

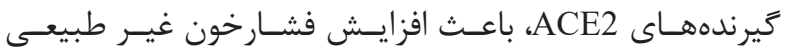

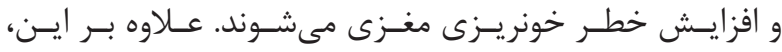

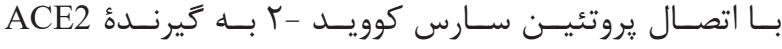

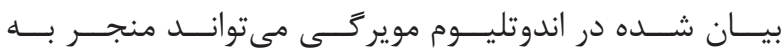

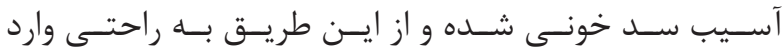

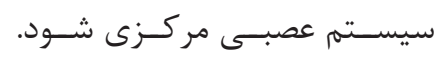

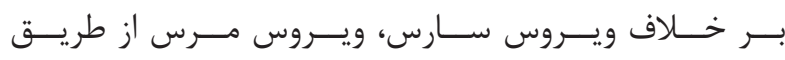

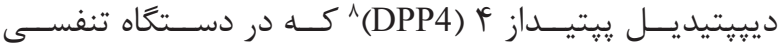

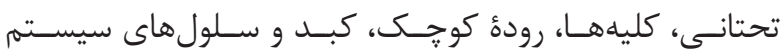

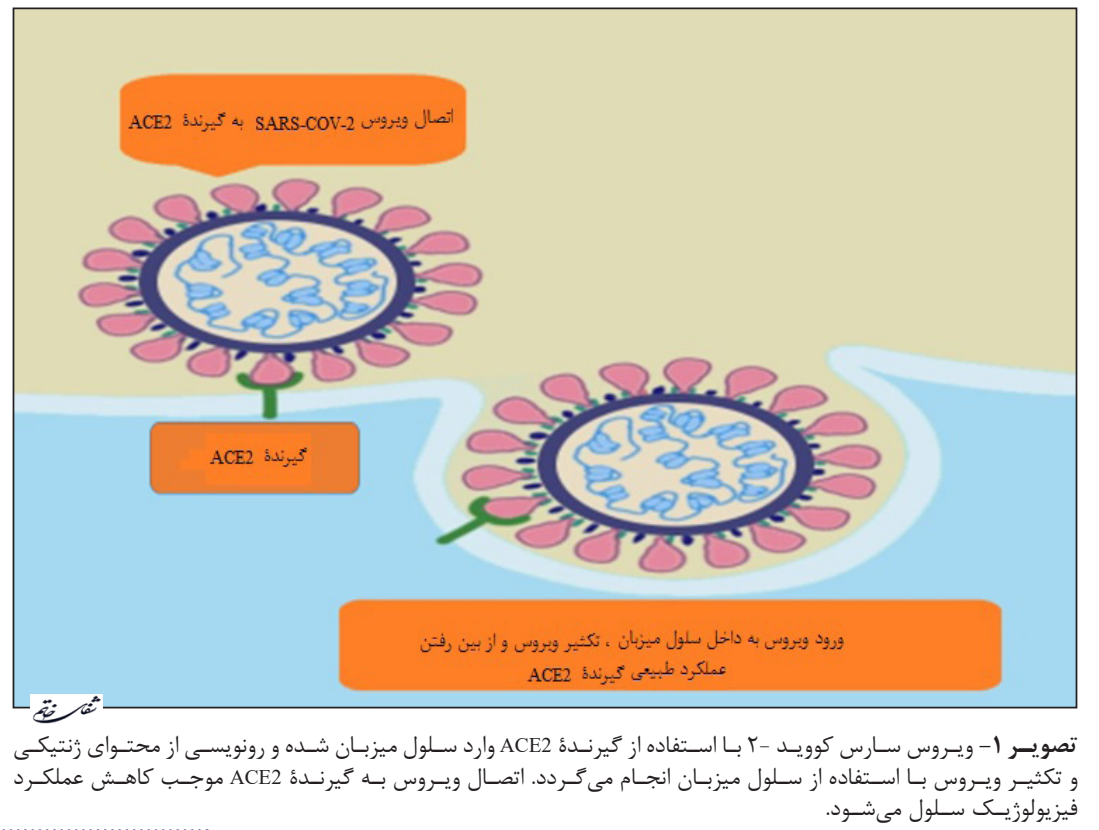

${ }^{7}$ Angiotensin-converting enzyme 2

${ }^{8}$ Dipeptidyl peptidase-4 


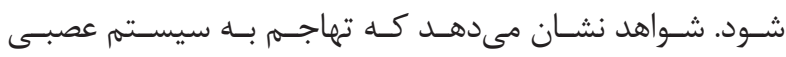

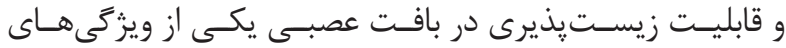

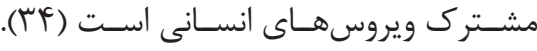
تشـخيص ميـزان بــالاى ويــروس در سـاقهُ مغـز زيسـ از ابتـلا

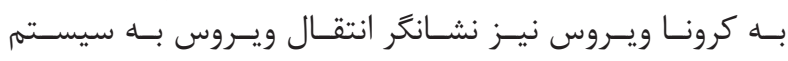

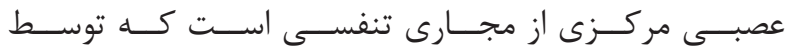

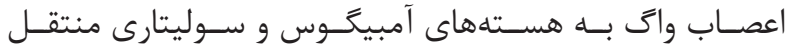

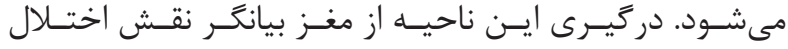

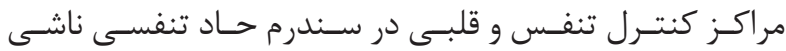

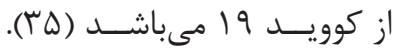

كسـترش ويروسهــا از محيــط بــه سيسـتم عصبـى مركـزى

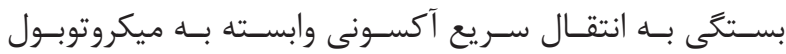

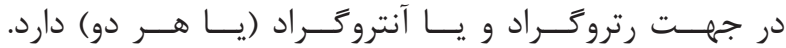

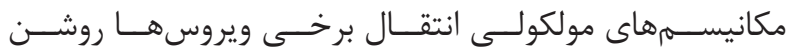

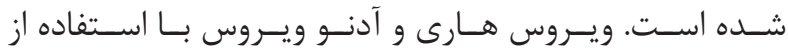

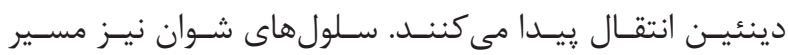

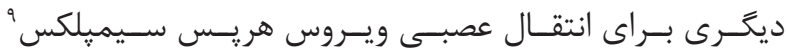

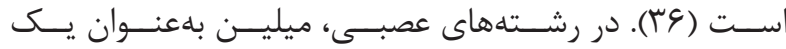

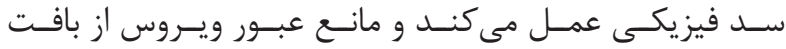

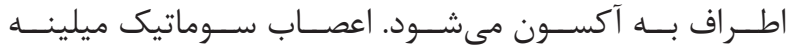

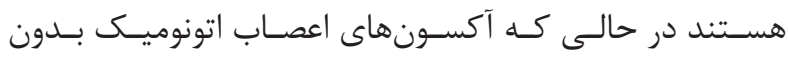

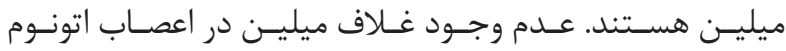

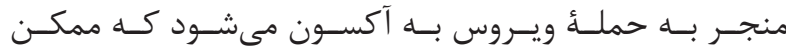

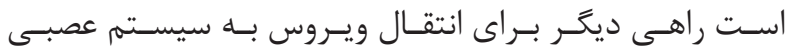

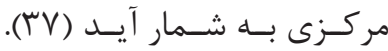

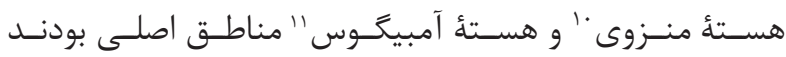

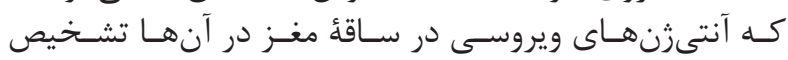

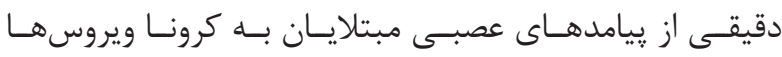

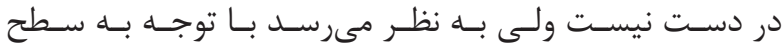

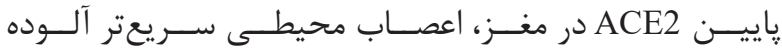

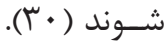

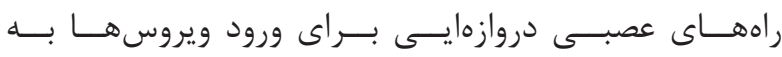

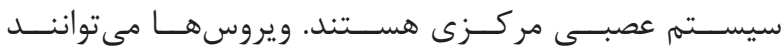

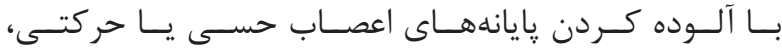

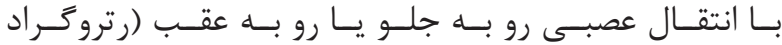

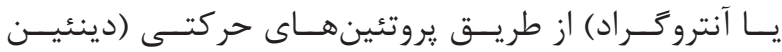

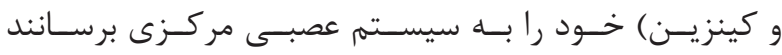

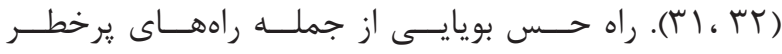

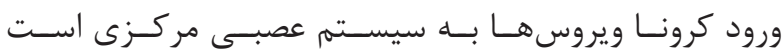

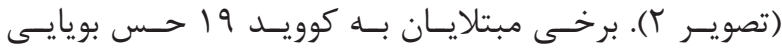

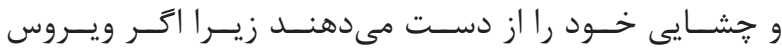

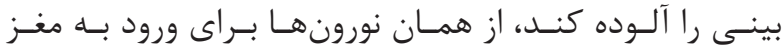

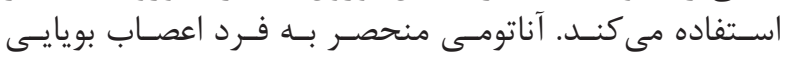

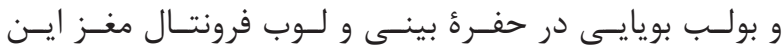

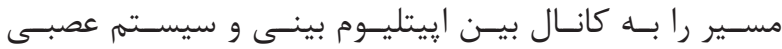

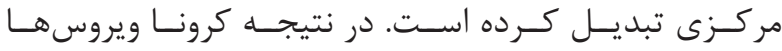

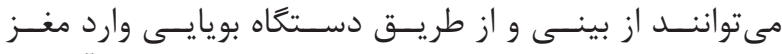

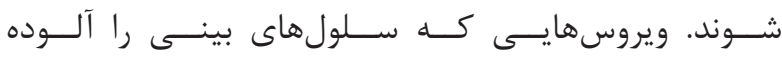

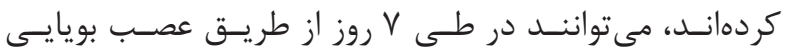

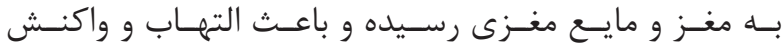

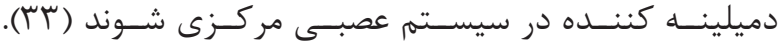

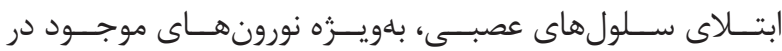

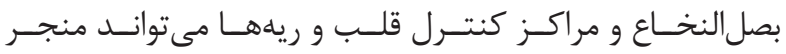

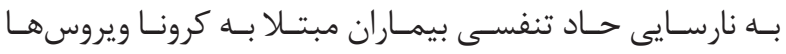

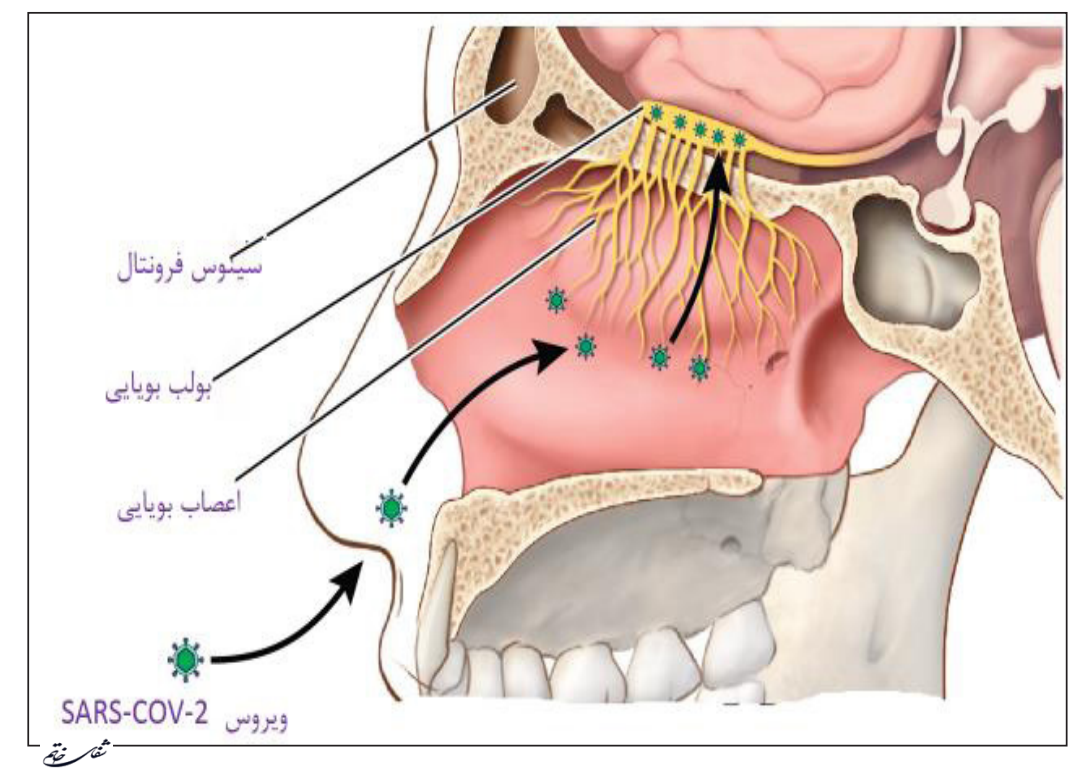

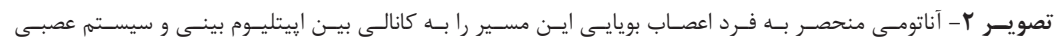

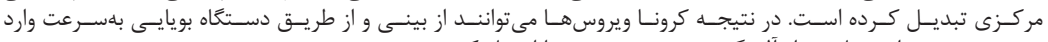

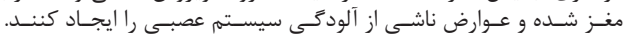

${ }^{9}$ Herpes simplex virus

${ }^{10}$ Solitary nucleus

${ }^{11}$ Nucleus ambiguus 


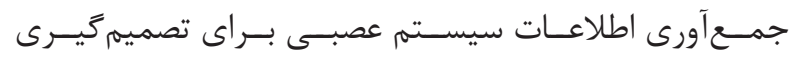

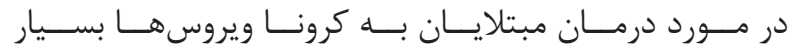

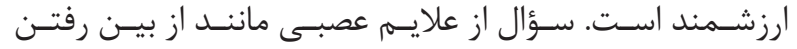

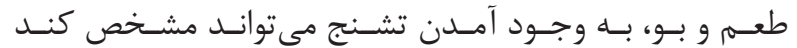

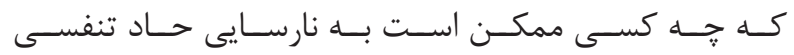

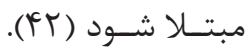

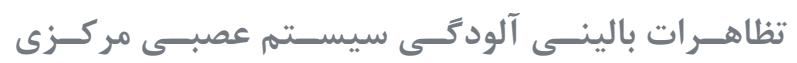

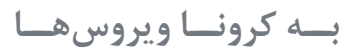

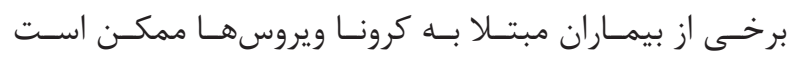

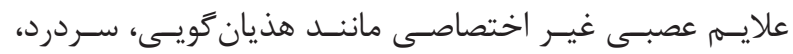

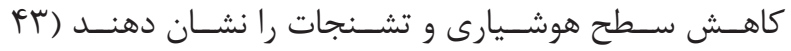

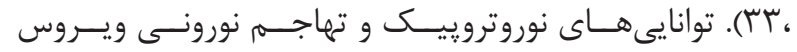

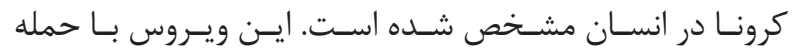

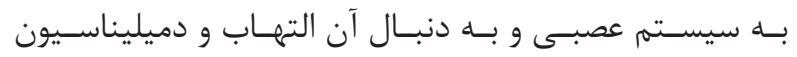

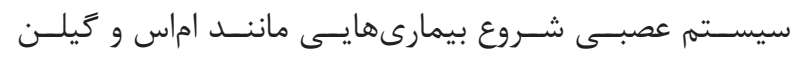

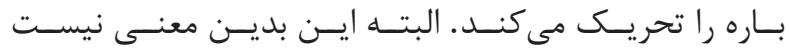

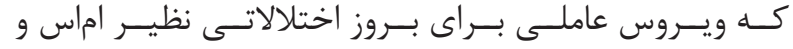

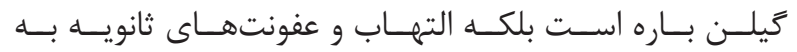

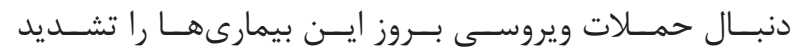

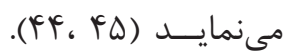

برخـى مبتلايـان نيـز تظاهـرات عصبـى را بـهـ دنبال مشـكلات

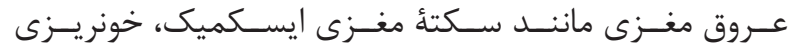

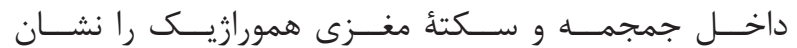

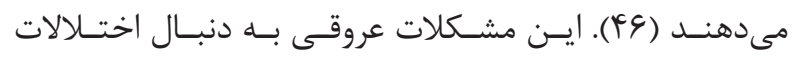

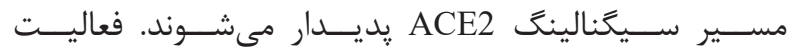

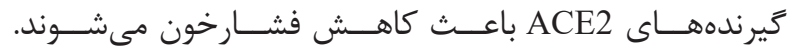

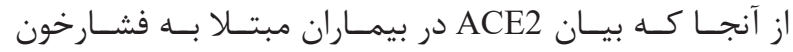

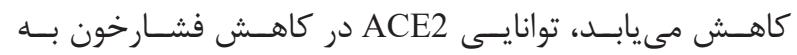

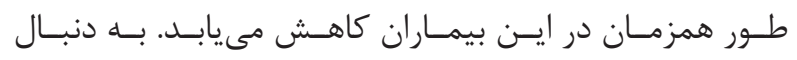

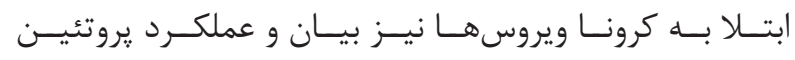

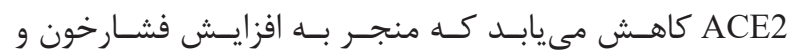

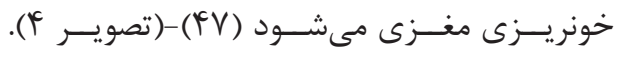

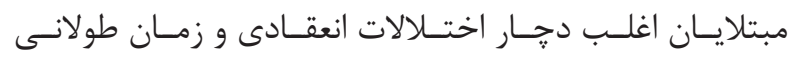

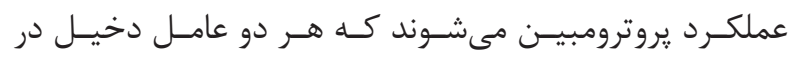

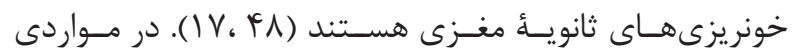

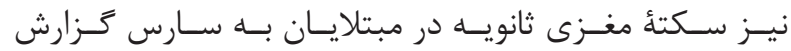

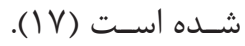

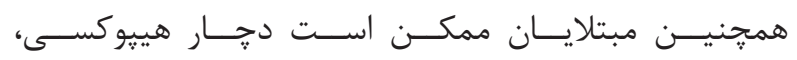

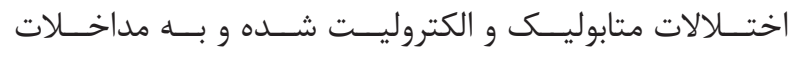

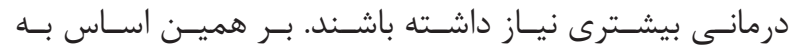

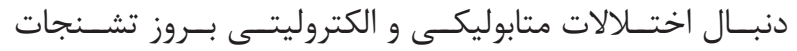

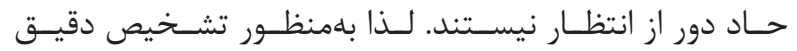

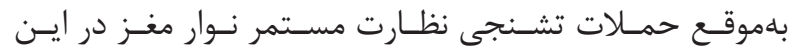

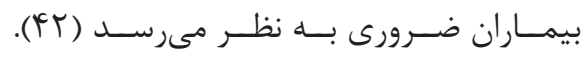

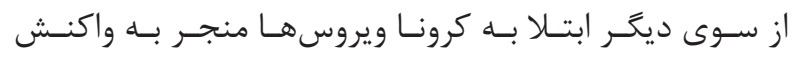

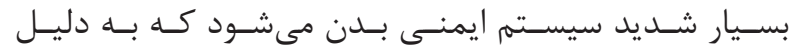

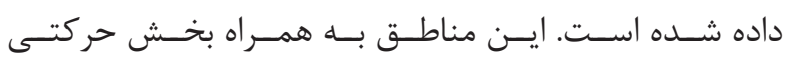

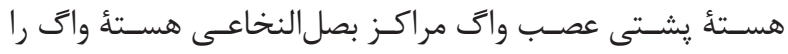

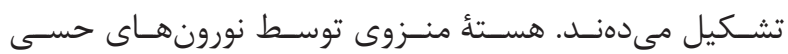

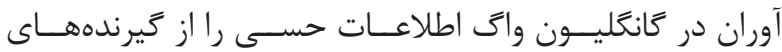

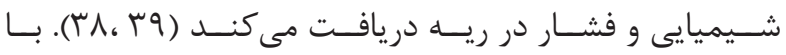

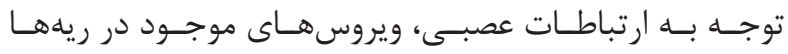

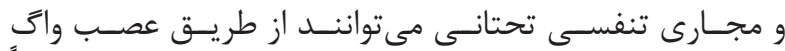

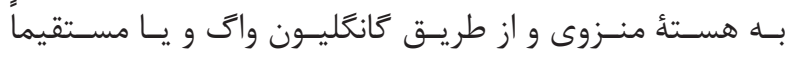

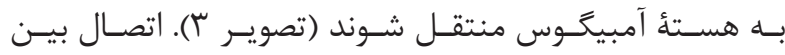

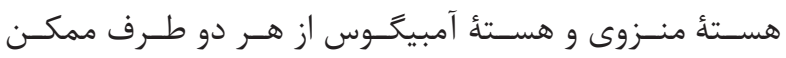

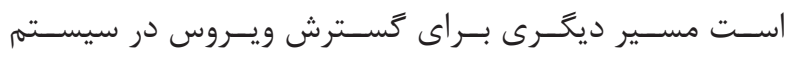

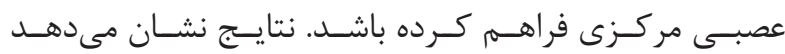

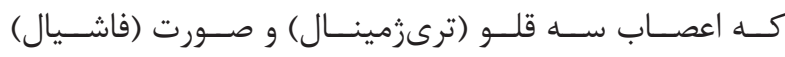

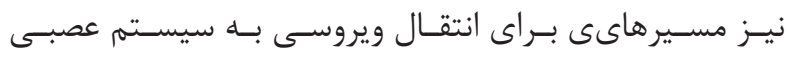

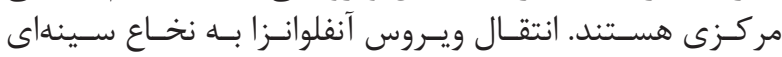

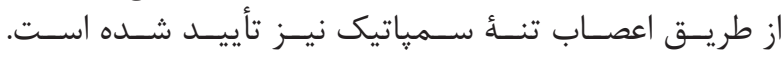

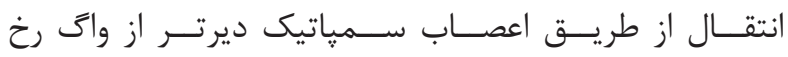

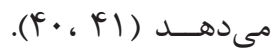

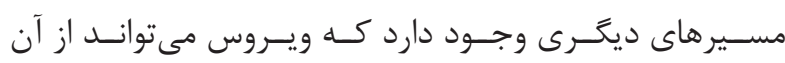

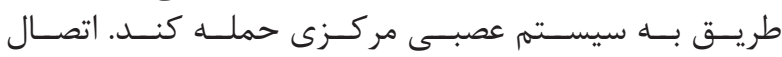

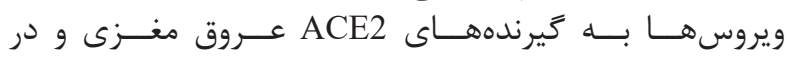

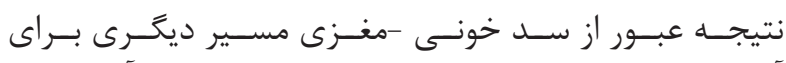

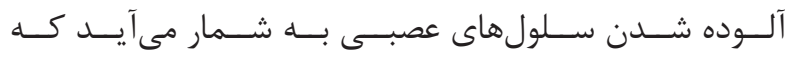

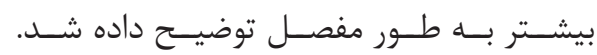

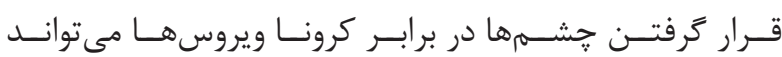

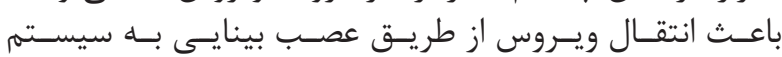

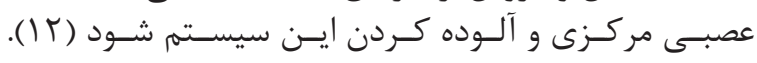

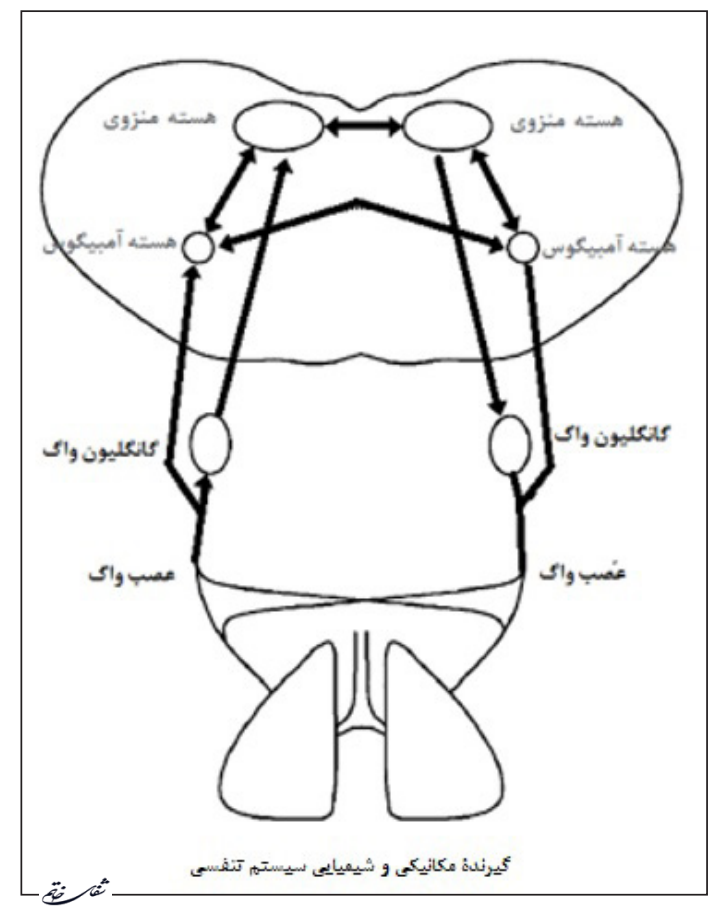

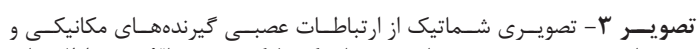

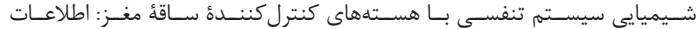

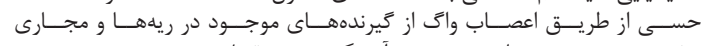

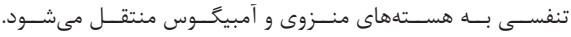




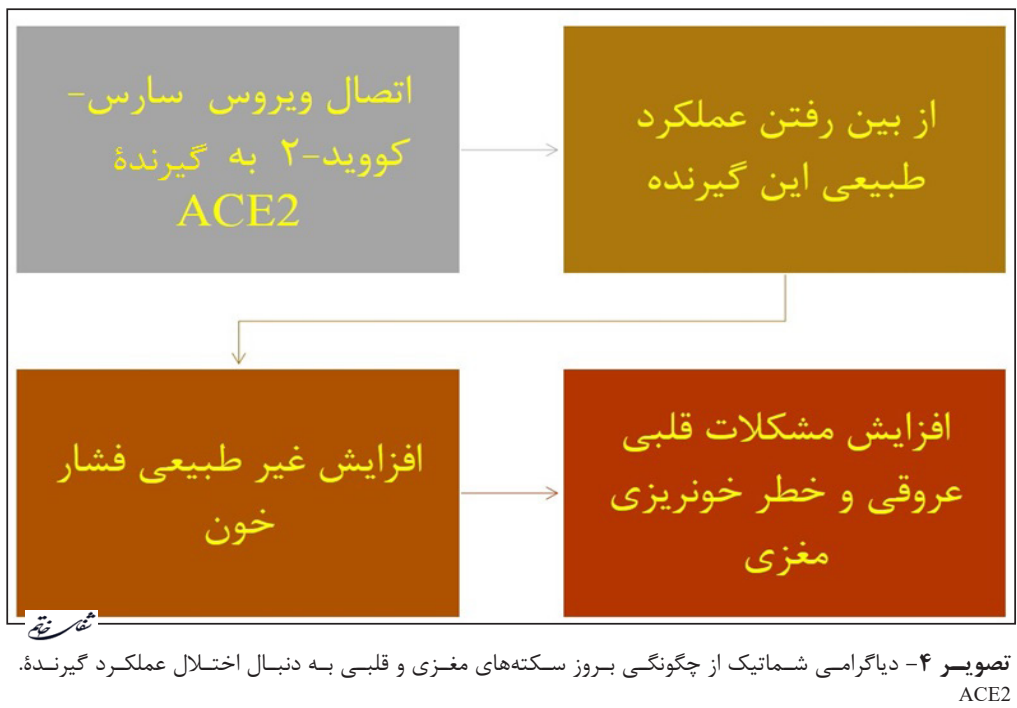

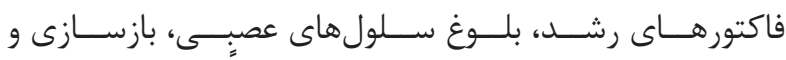

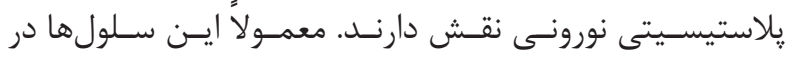

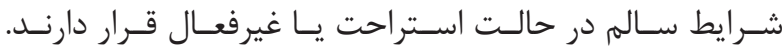

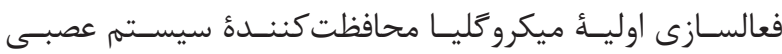

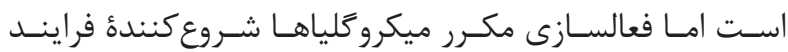

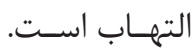

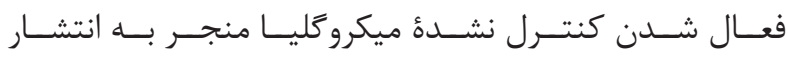
ســايتو كاين هاى التهابـى (IL-1B ، IL-6،TNF-

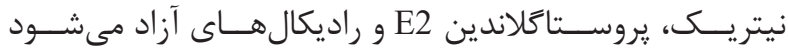

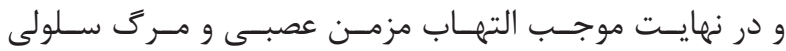

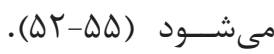

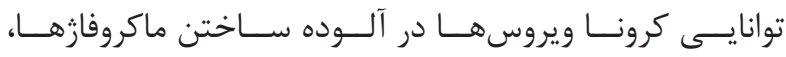

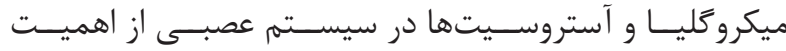

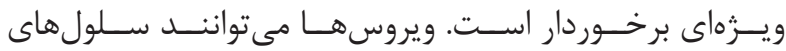

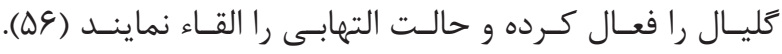

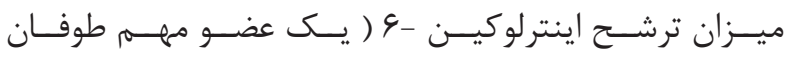

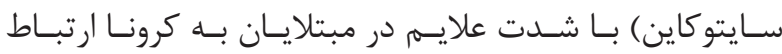

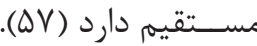

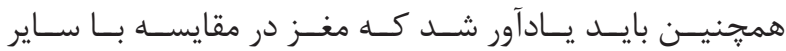

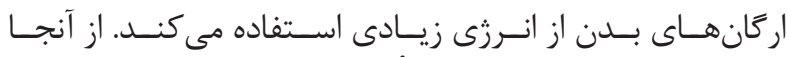

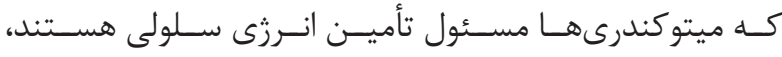

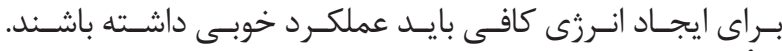

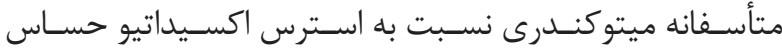

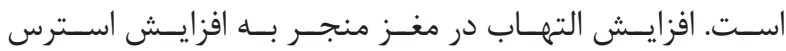

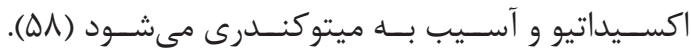

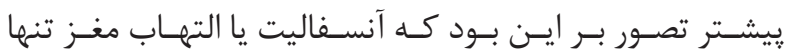

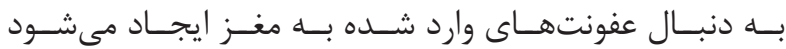

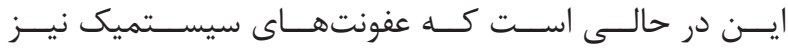

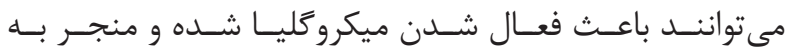

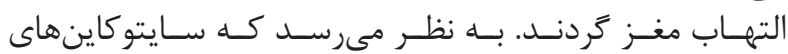

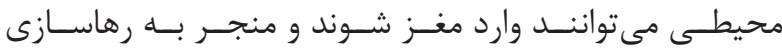

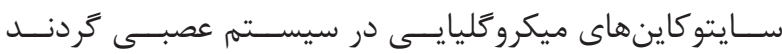

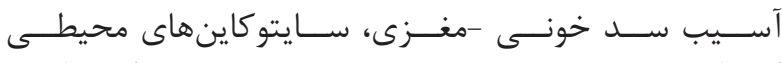

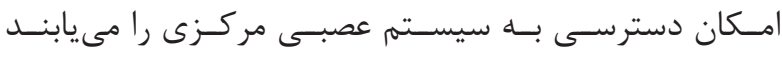

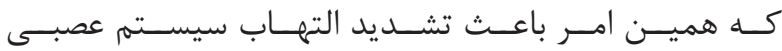
مى شـــود. بــه عبــارت ديخــر آسـيب سيسـتم عصبـى در مبتلايــان بــهـ

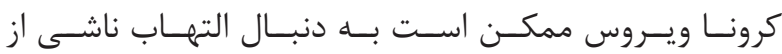

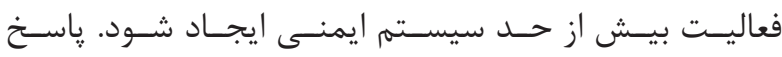

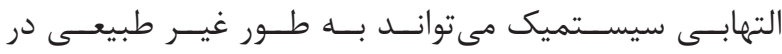

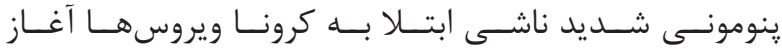

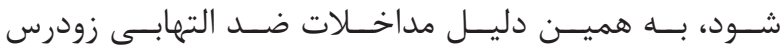

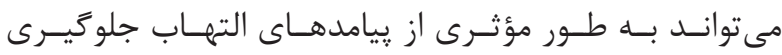

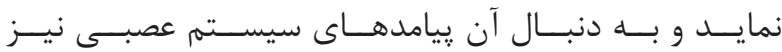

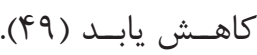

سيسـتم ايمنــى در سيســتم عصبــى مركــزى مانــــد ســاير

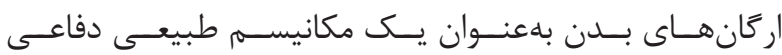

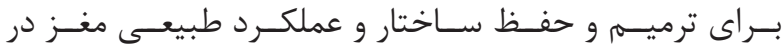

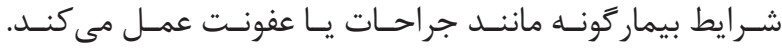

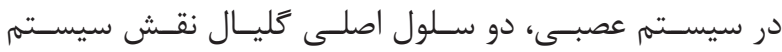

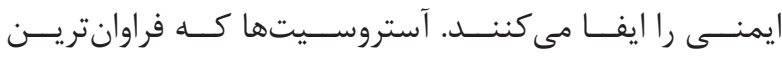

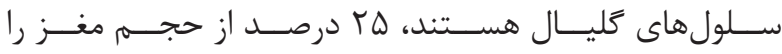

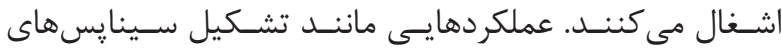

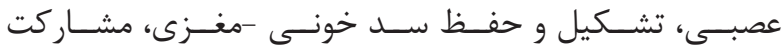

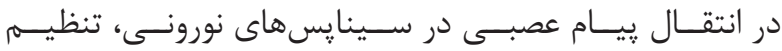

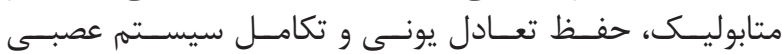

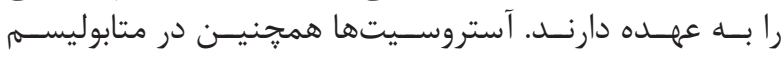

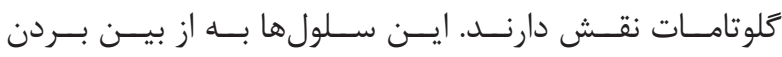

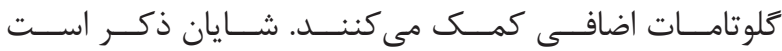

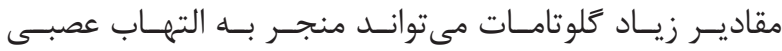

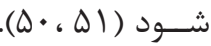

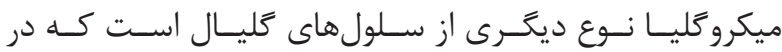

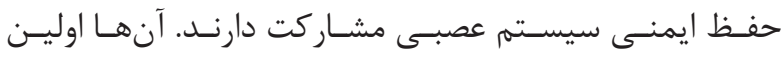

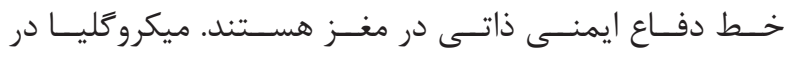

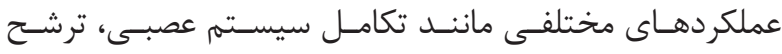




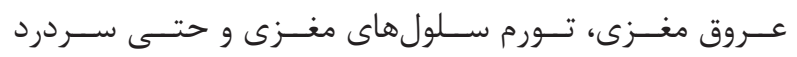

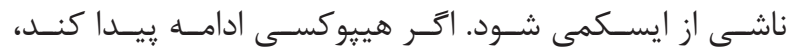

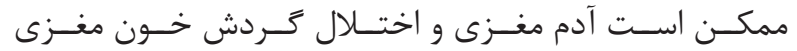

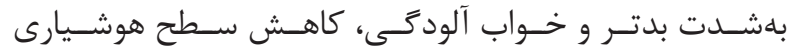

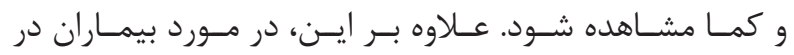

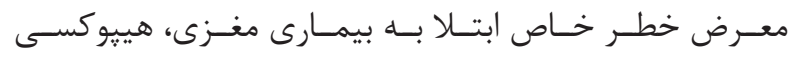

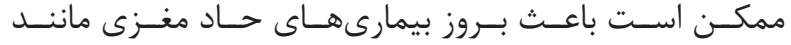

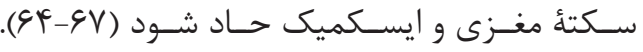

نتيجه

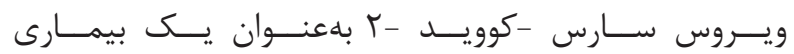

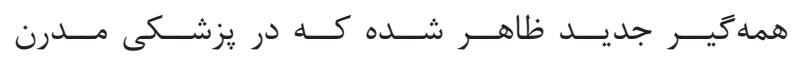

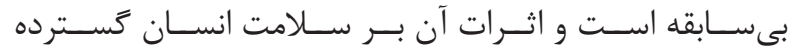

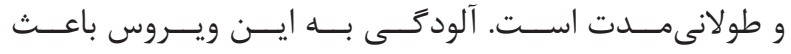

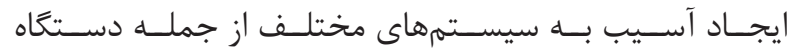

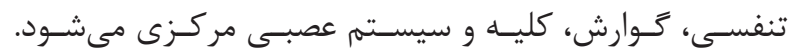

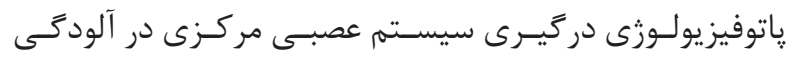

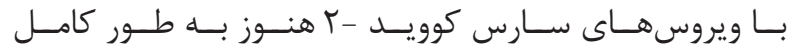

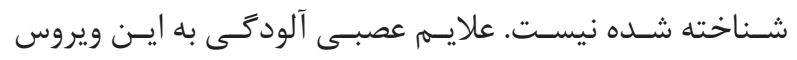

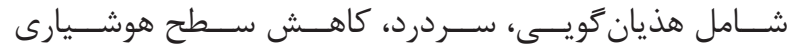

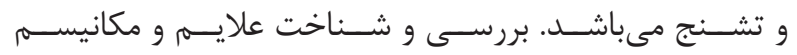

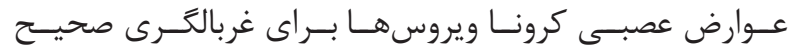

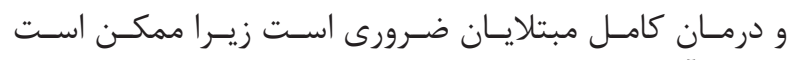

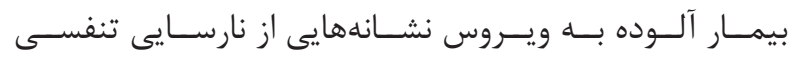

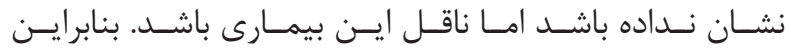

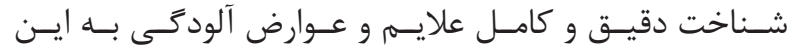

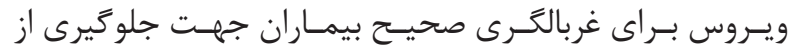

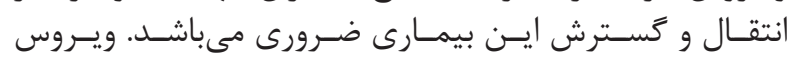

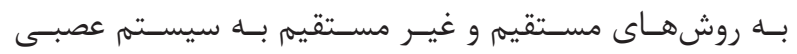

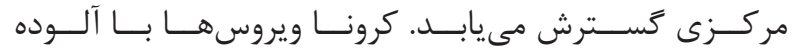

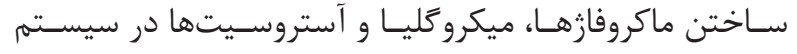

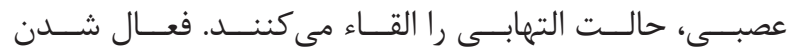

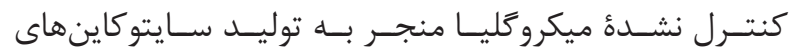

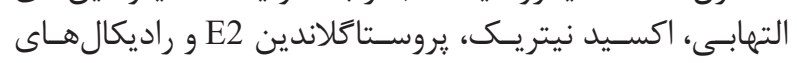

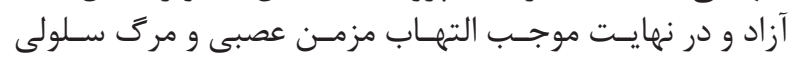

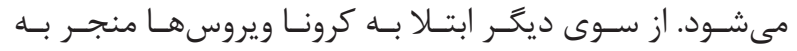

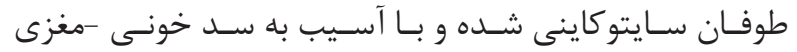

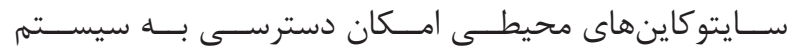

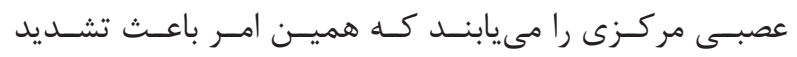

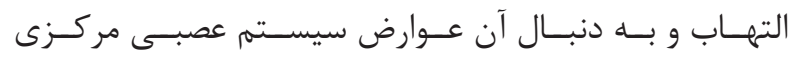

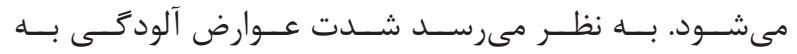

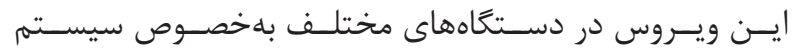

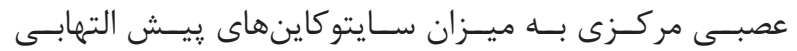

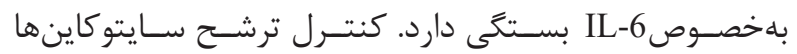

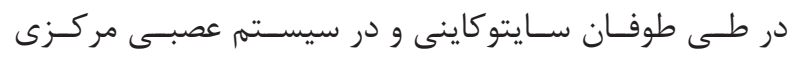

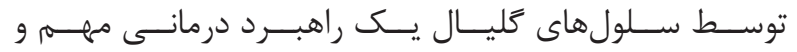

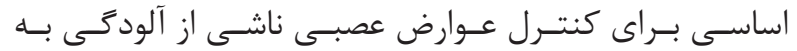

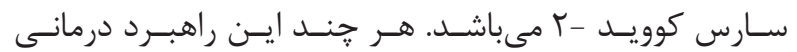

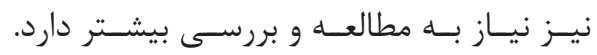

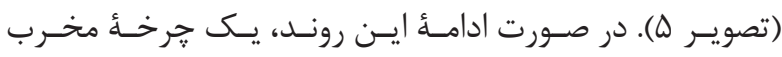

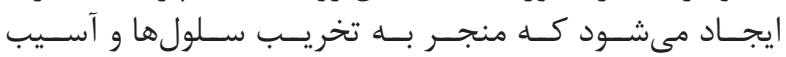

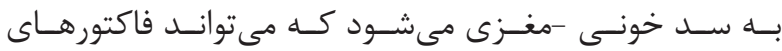
التهابـى بيشـترى راو وارد سيسـتمى عصبـى نمى نمايـد (ه9).

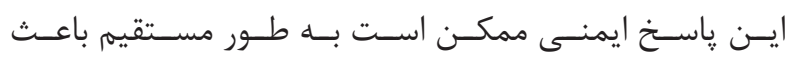

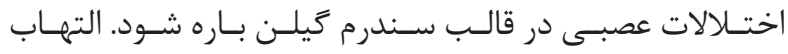

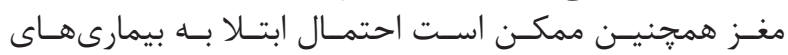

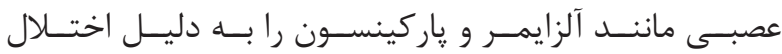

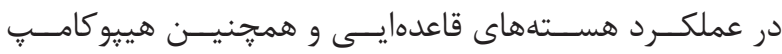

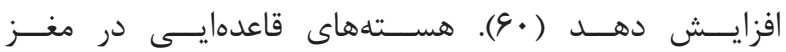

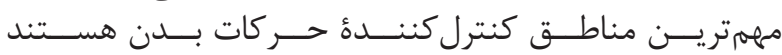

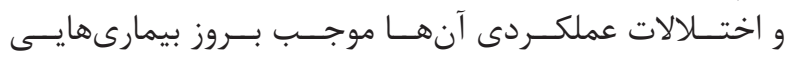

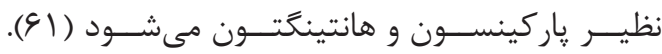

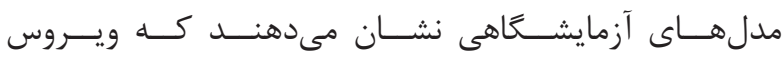

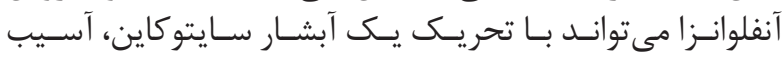

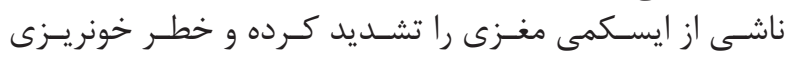

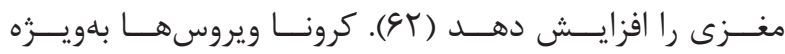

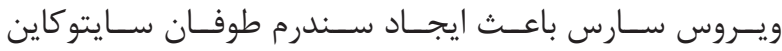

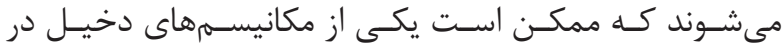

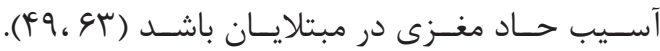

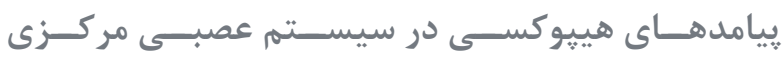

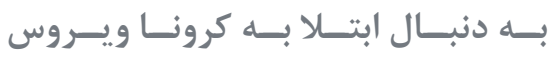

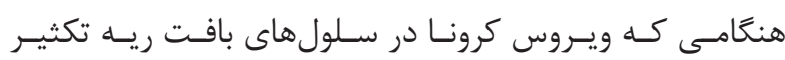

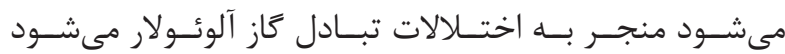

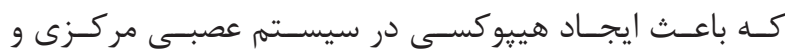

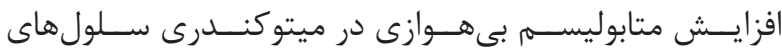

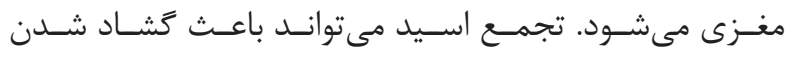

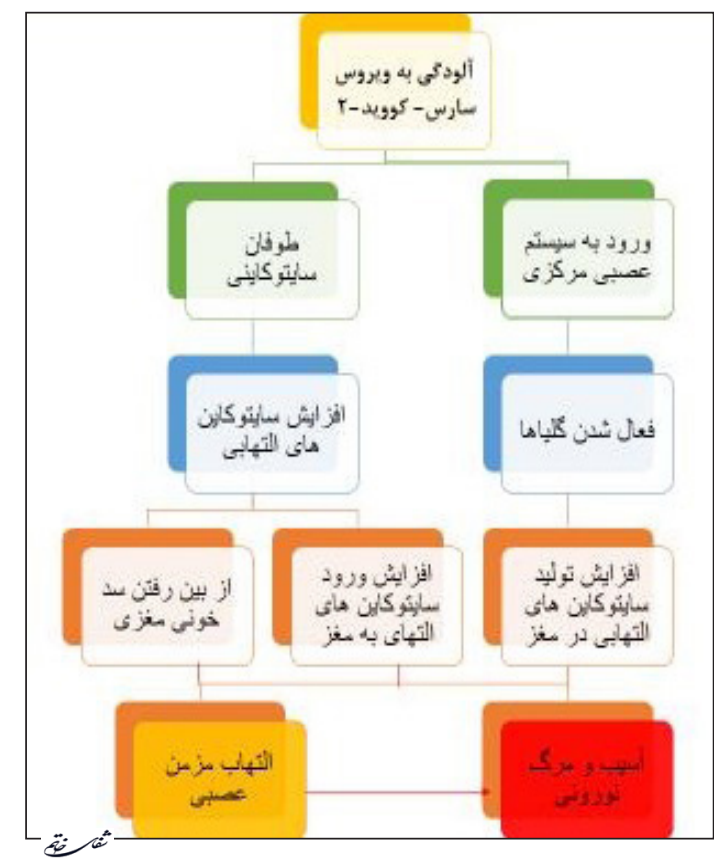

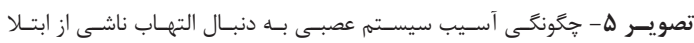

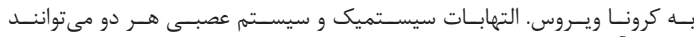

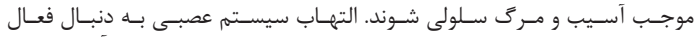

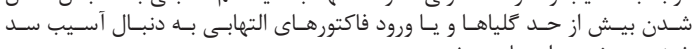
خونسي -منـزى ايجـاد مئشـود. 
1. Wu F, Zhao S, Yu B, Chen Y-M, Wang W, Song Z-G, et al. A new coronavirus associated with human respiratory disease in China. Nature. 2020; 579(7798): 265-9.

2. Su S, Wong G, Shi W, Liu J, Lai AC, Zhou J, et al. Epidemiology, genetic recombination, and pathogenesis of coronaviruses. Trends Microbiol. 2016; 24(6): 490502.

3. Wang W, Tang J, Wei F. Updated understanding of the outbreak of 2019 novel coronavirus (2019-nCoV) in Wuhan, China. J Med Virol. 2020; 92(4): 441-7.

4. Hopkins C, Kumar N. Loss of sense of smell as marker of COVID-19 infection. ENT UK [https:/www entuk org/sites/default/files/files/Loss of sense of smell as marker of COVID pdf] Date accessed. 2020; 26(03): 2020 .

5. Ren L-L, Wang Y-M, Wu Z-Q, Xiang Z-C, Guo L, $\mathrm{Xu} \mathrm{T}$, et al. Identification of a novel coronavirus causing severe pneumonia in human: a descriptive study. Chin Med J (Engl). 2020; 133(9): 1015-24.

6. Gu J, Han B, Wang J. COVID-19: gastrointestinal manifestations and potential fecal-oral transmission. Gastroenterology. 2020; 158(6): 1518-9.

7. Recalcati S. Cutaneous manifestations in COVID-19: a first perspective. J Eur Acad Dermatol Venereol. 2020; 34(5): e212-3.

8. Zhou Y, Duan C, Zeng Y, Tong Y, Nie Y, Yang Y, et al. Ocular findings and proportion with conjunctival SARS-COV-2 in COVID-19 patients. Ophthalmology. 2020; 127(7): 982-3.

9. Baud D, Qi X, Nielsen-Saines K, Musso D, Pomar L, Favre G. Real estimates of mortality following COVID-19 infection. Lancet Infect Dis. 2020; 20(7): 733. doi: 10.1016/S1473-3099(20)30195-X.

10. Fang L, Karakiulakis G, Roth M. Are patients with hypertension and diabetes mellitus at increased risk for COVID-19 infection? Lancet Respir Med. 2020; 8(4): e21. doi: 10.1016/S2213-2600(20)30116-8.

11. Carod-Artal F. Neurological complications of coronavirus and COVID-19. Rev Neurol. 2020; 70(9): 311-22.

12. Kotfis K, Williams Roberson S, Wilson JE, Dabrowski W, Pun BT, Ely EW. COVID-19: ICU delirium management during SARS-CoV-2 pandemic. Crit Care. 2020; 24(1): 176. doi: 10.1186/s13054-02002882-x.
13. Liu W, Li H. COVID-19: attacks the 1-beta chain of hemoglobin and captures the porphyrin to inhibit human heme metabolism. ChemRxiv. 2020; 10(04).

14. Danzi GB, Loffi M, Galeazzi G, Gherbesi E. Acute pulmonary embolism and COVID-19 pneumonia: a random association? Eur Heart J. 2020; 41(19): 1858. doi: 10.1093/eurheartj/ehaa254.

15. Mao L, Jin H, Wang M, Hu Y, Chen S, He Q, et al. Neurologic manifestations of hospitalized patients with coronavirus disease 2019 in Wuhan, China. JAMA Neurol. 2020; 77(6): 1-9.

16. Zhang Y, Xiao M, Zhang S, Xia P, Cao W, Jiang W, et al. Coagulopathy and antiphospholipid antibodies in patients with Covid-19. N Engl J Med. 2020; 382(17): e38. doi: 10.1056/NEJMc2007575.

17. Chen Z-M, Fu J-F, Shu Q, Chen Y-H, Hua C-Z, Li F-B, et al. Diagnosis and treatment recommendations for pediatric respiratory infection caused by the 2019 novel coronavirus. World J Pediatr. 2020; 16(3): 240-6.

18. Zheng Y-Y, Ma Y-T, Zhang J-Y, Xie X. COVID-19 and the cardiovascular system. Nat Rev Cardiol. 2020; 17(5): 259-60.

19. Rismanbaf A, Zarei S. Liver and kidney injuries in COVID-19 and their effects on drug therapy; a letter to editor. Arch Acad Emerg Med. 2020; 8(1): e17.

20. Xu L, Liu J, Lu M, Yang D, Zheng X. Liver injury during highly pathogenic human coronavirus infections. Liver Int. 2020; 40(5): 998-1004.

21. Zhang C, Shi L, Wang F-S. Liver injury in COVID-19: management and challenges. Lancet Gastroenterol Hepatol. 2020; 5(5): 428-30.

22. Donoghue M, Hsieh F, Baronas E, Godbout K, Gosselin M, Stagliano N, et al. A novel angiotensinconverting enzyme-related carboxypeptidase (ACE2) converts angiotensin I to angiotensin 1-9. Circ Res. 2000; 87(5): e1-e9.

23. Harmer D, Gilbert $M$, Borman R, Clark KL. Quantitative mRNA expression profiling of ACE 2, a novel homologue of angiotensin converting enzyme. FEBS Lett. 2002; 532(1-2): 107-10.

24. Hamming I, Timens W, Bulthuis M, Lely A, Navis $\mathrm{G}$, van Goor H. Tissue distribution of ACE2 protein, the functional receptor for SARS coronavirus. A first step in understanding SARS pathogenesis. J Pathol. 2004; 203(2): 631-7. 
25. Walls AC, Park Y-J, Tortorici MA, Wall A, McGuire AT, Veesler D. Structure, function, and antigenicity of the SARS-CoV-2 spike glycoprotein. Cell. 2020; 181(2): 281-92.

26. Mattern T, Scholz W, Feller A, Flad HD, Ulmer A. Expression of CD26 (dipeptidyl peptidase IV) on resting and activated human T-lymphocytes. Scand J Immunol. 1991; 33(6): 737-48.

27. Boonacker E, Van Noorden CJ. The multifunctional or moonlighting protein CD26/DPPIV. Eur J Cell Biol. 2003; 82(2): 53-73.

28. To K, Lo AW. Exploring the pathogenesis of severe acute respiratory syndrome (SARS): the tissue distribution of the coronavirus (SARS-CoV) and its putative receptor, angiotensin-converting enzyme 2 (ACE2). J Pathol. 2004; 203(3): 740-3.

29. Bernstein H-G, Dobrowolny H, Keilhoff G, Steiner J. Dipeptidyl peptidase IV, which probably plays important roles in Alzheimer disease (AD) pathology, is upregulated in $\mathrm{AD}$ brain neurons and associates with amyloid plaques. Neurochem Int. 2018; 114: 55-7.

30. Li YC, Bai WZ, Hashikawa T. The neuroinvasive potential of SARS-CoV2 may play a role in the respiratory failure of COVID-19 patients. J Med Virol. 2020; 92(6): 552-5.

31. Salinas S, Schiavo G, Kremer EJ. A hitchhiker's guide to the nervous system: the complex journey of viruses and toxins. Nat Rev Microbiol. 2010; 8(9): 645-55.

32. Swanson II PA, McGavern DB. Viral diseases of the central nervous system. Curr Opin Virol. 2015; 11: 44-54.

33. Bohmwald K, Galvez N, Ríos M, Kalergis AM. Neurologic alterations due to respiratory virus infections. Front Cell Neurosci. 2018; 12: 386. doi: 10.3389/fncel.2018.00386.

34. Baig AM, Khaleeq A, Ali U, Syeda H. Evidence of the COVID-19 virus targeting the CNS: tissue distribution, host-virus interaction, and proposed neurotropic mechanisms. ACS Chemical Neuroscience. 2020; 11(7): 995-8.

35. Sepehrinezhad A, Shahbazi A, Negah SS. COVID-19 virus may have neuroinvasive potential and cause neurological complications: a perspective review. J Neurovirol. 2020; 26(3): 324-9.

36. Shimeld C, Efstathiou S, Hill T. Tracking the spread of a lacZ-tagged herpes simplex virus type 1 between the eye and the nervous system of the mouse: comparison of primary and recurrent infection. J Virol. 2001; 75(11): 5252-62.

37. Hill TJ. Ocular pathogenicity of herpes simplex virus. Curr Eye Res. 1987; 6(1): 1-7.

38. Hadziefendic S, Haxhiu MA. CNS innervation of vagal preganglionic neurons controlling peripheral airways: a transneuronal labeling study using pseudorabies virus. J Auton Nerv Syst. 1999; 76(2-3): 135-45.

39. Kalia M, Mesulam MM. Brain stem projections of sensory and motor components of the vagus complex in the cat: II. Laryngeal, tracheobronchial, pulmonary, cardiac, and gastrointestinal branches. J Comp Neurol. 1980; 193(2): 467-508.

40. Park C, Ishinaka M, Takada A, Kida H, Kimura T, Ochiai $\mathrm{K}$, et al. The invasion routes of neurovirulent A/Hong Kong/483/97 (H5N1) influenza virus into the central nervous system after respiratory infection in mice. Arch Virol. 2002; 147(7): 1425-36.

41. Matsuda K, Park C, Sunden Y, Kimura T, Ochiai K, $\mathrm{Kida} \mathrm{H}$, et al. The vagus nerve is one route of transneural invasion for intranasally inoculated influenza a virus in mice. Vet Pathol. 2004; 41(2): 101-7.

42. Karimi N, Sharifi Razavi A, Rouhani N. Frequent convulsive seizures in an adult patient with COVID-19: a case report. Iranian Red Crescent Medical Journal. 2020.

43. Desforges M, Le Coupanec A, Dubeau P, Bourgouin A, Lajoie L, Dubé M, et al. Human coronaviruses and other respiratory viruses: underestimated opportunistic pathogens of the central nervous system? Viruses. 2020; 12(1): 14. doi: 10.3390/v12010014.

44. Lane TE, Buchmeier MJ. Murine coronavirus infection: a paradigm for virus-induced demyelinating disease. Trends Microbiol. 1997; 5(1): 9-14.

45. Virani A, Rabold E, Hanson T, Haag A, Elrufay R, Cheema $\mathrm{T}$, et al. Guillain-Barré syndrome associated with SARS-CoV-2 infection. IDCases. 2020: e00771. doi: 10.1016/j.idcr.2020.e00771.

46. Dogra S, Jain R, Cao M, Bilaloglu S, Zagzag D, Hochman S, et al. Hemorrhagic stroke and anticoagulation in COVID-19. J Stroke Cerebrovasc Dis. 2020; 29(8): 104984.

47. Schiffrin EL, Flack JM, Ito S, Muntner P, Webb RC. Hypertension and COVID-19. Oxford University Press US; 2020.

48. Wang D, Hu B, Hu C, Zhu F, Liu X, Zhang J, et al. 
Clinical characteristics of 138 hospitalized patients with 2019 novel coronavirus-infected pneumonia in Wuhan, China. JAMA. 2020; 323(11): 1061-9.

49. Mehta P, McAuley DF, Brown M, Sanchez E, Tattersall RS, Manson JJ. COVID-19: consider cytokine storm syndromes and immunosuppression. Lancet. 2020; 395(10229): 1033-4.

50. Arcuri C, Mecca C, Bianchi R, Giambanco I, Donato $\mathrm{R}$. The pathophysiological role of microglia in dynamic surveillance, phagocytosis and structural remodeling of the developing CNS. Front Mol Neurosci. 2017; 10: 191. doi: 10.3389/fnmol.2017.00191.

51. Yin J, Valin KL, Dixon ML, Leavenworth JW. The role of microglia and macrophages in CNS homeostasis, autoimmunity, and cancer. J Immunol Res. 2017; 2017. doi: $10.1155 / 2017 / 5150678$.

52. Vargas DL, Nascimbene C, Krishnan C, Zimmerman AW, Pardo CA. Neuroglial activation and neuroinflammation in the brain of patients with autism. Ann Neurol. 2005; 57(1): 67-81.

53. Block ML, Calderón-Garcidueñas L. Air pollution: mechanisms of neuroinflammation and CNS disease Trends Neurosci. 2009; 32(9): 506-16.

54. Wang W-Y, Tan M-S, Yu J-T, Tan L. Role of proinflammatory cytokines released from microglia in Alzheimer's disease. Ann Transl Med. 2015; 3(10): 136. doi: $\quad 10.3978 /$ j.issn.2305-5839.2015.03.49.

55. Smith JA, Das A, Ray SK, Banik NL. Role of pro-inflammatory cytokines released from microglia in neurodegenerative diseases. Brain Res Bull. 2012; 87(1): $10-20$.

56. Li Y, Fu L, Gonzales DM, Lavi E. Coronavirus neurovirulence correlates with the ability of the virus to induce proinflammatory cytokine signals from astrocytes and microglia. J Virol. 2004; 78(7): 3398-406.

57. Wan S, Yi Q, Fan S, Lv J, Zhang X, Guo L, et al. Characteristics of lymphocyte subsets and cytokines in peripheral blood of 123 hospitalized patients with 2019 novel coronavirus pneumonia (NCP). MedRxiv. 2020. doi: 10.1101/2020.02.10.20021832.

58. Onyango IG, Khan SM, Bennett Jr JP. Mitochondria in the pathophysiology of Alzheimer's and Parkinson's diseases. Front Biosci (Landmark Ed). 2017; 22: 854-72. 59. Banks WA, Kastin AJ. Blood to brain transport of interleukin links the immune and central nervous systems. Life Sci. 1991; 48(25): PL117-PL21.

60. Heneka MT, Carson MJ, El Khoury J, Landreth GE, Brosseron F, Feinstein DL, et al. Neuroinflammation in Alzheimer's disease. Lancet Neurol. 2015; 14(4): 388405 .

61. Hirsch EC, Vyas S, Hunot S. Neuroinflammation in Parkinson's disease. Parkinsonism \& Related Disorders. 2012; 18: S210-S2.

62. Muhammad S, Haasbach E, Kotchourko M, Strigli A, Krenz A, Ridder DA, et al. Influenza virus infection aggravates stroke outcome. Stroke. 2011; 42(3): 783-91.

63. Chen C, Zhang X, Ju Z, He W. Advances in the research of cytokine storm mechanism induced by Corona Virus Disease 2019. Zhonghua Shao Shang Za Zhi. 2020; 36(6): 471-5.

64. Guo Y-R, Cao Q-D, Hong Z-S, Tan Y-Y, Chen S-D, Jin $\mathrm{H}-\mathrm{J}$, et al. The origin, transmission and clinical therapies on coronavirus disease 2019 (COVID-19) outbreak-an update on the status. Mil Med Res. 2020; 7(1): 11. doi: 10.1186/s40779-020-00240-0.

65. Kashani KB. Hypoxia in COVID-19: Sign of Severity or Cause for Poor Outcomes. Mayo Clin Proc. 2020; 95(6): 1094-6.

66. Xu Z, Shi L, Wang Y, Zhang J, Huang L, Zhang C, et al. Pathological findings of COVID-19 associated with acute respiratory distress syndrome. Lancet Respir Med. 2020; 8(4): 420-2.

67. Wu Y, Xu X, Chen Z, Duan J, Hashimoto K, Yang L, et al. Nervous system involvement after infection with COVID-19 and other coronaviruses. Brain Behav Immun. 2020; 87: 18-22. 